\title{
In-medium Hadron Modification and Meson Spectroscopy at CLAS
}

\author{
Chaden Djalali ${ }^{1}$ \\ University of South Carolina \\ Department of Physics and Astronomy, 712 Main Street, Columbia, Sc 29208, USA \\ E-mail: djalaliesc.edu
}

\section{Dennis Weygand}

Thomas Jefferson National Accelerator Facility

12000 Jefferson Avenue, Newport News, VA 23606, USA

E-mail: weygandejlab.org

\begin{abstract}
The theory of the strong interaction, Quantum Chromodynamics (QCD), has been remarkably successful in describing high-energy and short-distance-scale experiments involving quarks and gluons. However, applying QCD to low energy and large-distance-scale experiments has been a major challenge. At low energies, standard nuclear physics with nucleon and meson degrees of freedom work effectively in describing observations. The transition between the two descriptions of hadrons is one of the main goals of hadronic physics. Lattice QCD calculations have made tremendous progress and will one day give us a full description of strong interactions under all regimes. Meanwhile, effective theories incorporating some of the main features of QCD have been successfully developed and help us gain insight into the non-perturbative regime of QCD. All these models predict modifications of the properties of hadrons embedded in nuclear matter. Mesons with exotic quantum numbers are predicted at excitation energies below $2 \mathrm{GeV}$. Several experiments have been conducted with the Cebaf Large Acceptance Spectrometer (CLAS) at the Thomas Jefferson National Laboratory (JLab) to test these predictions.
\end{abstract}

XVIII International Workshop on Deep-Inelastic Scattering and Related Subjects - DIS 2010

Firenze, Italy

April 19-232010

1 Speaker 


\section{Introduction}

The study of the properties of hadrons in the medium as well as the search for exotic mesons provide stringent tests for hadronic models describing the non-pertubative regime of QCD. Chiral symmetry is predicted to be restored at high temperature and/or densities. In ordinary nuclear matter, the value of the quark condensate is predicted to drop by $35 \%$. The inmedium properties of light vector mesons $(\rho, \omega$ and $\varphi$ ) have been intensively studied in many experiments ranging from heavy-ion collisions to elementary reactions with $\gamma, \pi$ and $p$.

QCD models of hadrons have long suggested the existence of mesons beyond the Naïve Quark Model (NQM). Recent QCD calculations on the lattice ${ }^{1}$ confirm that there should exist mesonic states with quantum numbers explicitly prohibited by NQM, while QCD inspired models have also suggested that the production of such states may be enhanced in photoproduction reactions.

\section{Vector mesons in the medium.}

Several comprehensive reviews have been written about the medium modifications of the properties of vector mesons in the medium. ${ }^{2,3}$ QCD sum rules in the medium predict that the mass of the vector mesons drops linearly with the density. A drop of $\approx 15 \%$ in the mass of the $\rho$-meson is predicted at normal nuclear density. ${ }^{4}$ Hadronic models taking into account the in medium self energy of the meson predict non-trivial modifications of the spectral functions (spectral shifts, spectral broadening and new spectral peaks). ${ }^{\mathbf{5}}$

Many experiments have been studying the properties of the light vector mesons $\rho, \omega$ and $\phi$ in the medium, using simple probes such as $\gamma, \pi$ or $p$ on nuclei ( $T=0$ and $\rho \sim \rho_{0}$ ) or in relativistic heavy ion collisions ( $\mathrm{T}$ and/or $\rho>0$ ). The $\rho$ meson with its very short lifetime has a large probability of decaying in the medium. It is the best candidate for observing any medium modification by measuring its in medium mass spectrum. The $\omega$ and $\varphi$ mesons mostly decay outside the medium in which they are produced making it very difficult to directly measure any medium modification. Their in medium width can however be determine through transparency ratios. The most interesting property of these vector mesons is that they decay into di-leptons, which have negligible final state interactions. However, measuring the di-lepton spectra is very challenging because of the very small branching ratio $\left(\sim 10^{-5}\right)$.

The CLAS experiment has measured the photoproduction of the vector mesons in several nuclei. No mass shift is observed but a substantial broadening of the $\rho$-meson consistent with collisional broadening in the medium. ${ }^{6,7}$ These results are consistent with the observations reported by heavy ion experiments. ${ }^{\mathbf{8} 9}$ The $\omega$ and $\varphi$ meson widths deduced from transparencyratio measurements are factors of 5 to 15 times larger than in the vacuum. ${ }^{\mathbf{1 0}}$ The majority of experiments do not find evidence for mass shifts in the medium. The latest models predict that most of the medium modifications happen when the meson have momenta less than $0.7 \mathrm{GeV} / \mathrm{c}$ with respect to the medium. Next generation experiments with much higher statistics and improved acceptance for low momenta mesons are needed to obtain reliable results. Several experiments are planned at JPARC, JLab, RHIC, FAIR and CERN. During the next decade, 
substantial theoretical and experimental efforts will continue to be being carried out in this very active field.

\section{Meson Spectroscopy.}

Mesons are the simplest quark bound state, i.e. the best benchmark to understand how quarks interact to form hadrons and what the role of gluons is. The search for unusual states as hybrids (qqg), tetraquarks (qqq) and glueballs has been going on for some time. Of particular interest are mesons whose quantum numbers are excluded by the NQM. In particular, the fluxtube model suggests that the lowest lying hybrid state with manifestly exotic quantum numbers would be a $\pi_{1}$ meson with $\mathrm{J}^{\mathrm{PC}}=1^{-+}$. Isgur et al., ${ }^{11}$ suggested that such a state would be copiously produced in diffractive photoproduction, as one merely had to 'pluck' the flux tube of the unexcited vector dominance $\rho$ component of the incident photon to excite it into a $\pi_{1}$ exotic mode.

To date, three candidates for mesons with $\mathrm{J}^{\mathrm{PC}}=1^{-+}$and isospin 1 , have been reported experimentally: the $\pi_{1}(1400)$, the $\pi_{1}(1600)$, and the $\pi_{1}(2010)$. The $\pi_{1}(1400)$ was first observed ${ }^{12}$ by E852 experiment at the Brookhaven National Laboratory (BNL), and subsequently seen at the Crystal Barrel in anti-proton neutron annihilations. ${ }^{13}$

The best candidate for the existence of mesons outside of the NQM is the $\pi_{1}(1600)$. This state was also observed by the E852 collaboration in the decay to $\rho \pi .{ }^{14}$ While the signal for the $\pi_{1}(1600)$ is not strong, the state is rather clearly seen by its interference with the wellestablished $\pi_{2}(1670)$, and represents about $4 \%$ of the cross section relative to the $a_{2}(1320)$. While some recent analysis of the E852 results created some controversy, ${ }^{15}$ more recent results from the COMPASS Collaboration ${ }^{16}$ clearly resolve the issue in favor of the original E852 claim. The $\pi_{1}(1600)$ is seen most clearly, however, through its decay to $\eta$ ' $\pi$ in the E852 data. Here the state may be seen directly in an invariant mass plot, while the PWA identifies it clearly as $\mathrm{J}^{\mathrm{PC}}=1^{-+}$.

The first attempt to search for exotic mesons in photoproduction experiments, ${ }^{17}$ inspired by the conjecture of Isgur and Paton, was carried out by the SLAC Hybrid Facility Photon Collaboration, which reported an isovector state at $1775 \mathrm{MeV} / \mathrm{c}^{2}$ decaying into three pions, and consistent with $\mathrm{J}^{\mathrm{PC}}=1^{-+}$, however, the data was also consistent with the non-exotic $\mathrm{J}^{\mathrm{PC}}=2^{-+}$ $\pi_{2}(1670)$.

Ideal conditions such as high intensity photon beams, and a large acceptance detector, are found at JLab. Several photoproduction experiments using CLAS have been carried out to look for exotic mesons. In 2001, a search for exotic mesons photoproduced by the charge exchange reaction $\gamma \mathrm{p} \rightarrow \pi^{+} \pi^{+} \pi$ - (n) was carried out by the CLAS collaboration (g6 experiment). A tagged-photon beam with energies in the 4.8 to $5.4 \mathrm{GeV}$ range, produced through bremsstrahlung from a $5.744 \mathrm{GeV}$ electron beam, was incident on a liquid-hydrogen target. The total raw luminosity was $2.7 \mathrm{pb}^{-1}$. A Partial Wave Analysis (PWA) was performed on a sample of 83,000 events, the highest such statistics to date in this reaction at these energies. ${ }^{18}$

Clear evidence for baryon resonance production is seen in the effective mass spectra of $n$ $\pi^{-}$, and $\mathrm{n} \pi^{+}$. The background was reduced by kinematic cuts on the data and the production of the $a_{2}(1320)$ is clearly observed together with a second structure above $1600 \mathrm{MeV}$. The two 
most prominent features of the extensive PWA results are the $\mathrm{J}^{\mathrm{PC}}=2^{++}$wave, consistent with the $\mathrm{a}_{2}(1320)$, and the $\mathrm{J}^{\mathrm{PC}}=2^{-+}$wave, consistent with the $\pi_{2}(1670)$, and seen in both the $\rho$ and $\mathrm{f}_{2}$ isobars. In all likelihood, the isovector meson reported at $1775 \mathrm{MeV}$ by the SLAC Hybrid Facility Photon Collaboration, was the $\pi_{2}(1670)$. The exotic $\pi_{1}(1600)$ is not seen in the PWA, and an upper limit on the cross section times branching ratio of 13.5 nbarns was determined, which is less than $2 \%$ of the $\mathrm{a}_{2}(1320)$. If the $\pi_{1}(1600)$ is primarily an hybrid, it would suggest that photoproduction does not favor $\mathrm{J}^{\mathrm{PC}}=1^{-+}$.

A second high-energy, high luminosity, photon run was initiated in the CLAS detector in 2009 (g12 experiment). The tagged-photon beam had energies between 4.4 to $5.4 \mathrm{GeV}$ range, produced through bremsstrahlung from a $5.744 \mathrm{GeV}$ electron beam, was incident on a $40 \mathrm{~cm}$ liquid-hydrogen target. The total raw luminosity was $27 \mathrm{pb}^{-1}, 10$ times the original $\mathrm{g} 6$ experiment. The calibration and reconstruction phase ended in January 2010, and spin-parity analysis is proceeding. In this experiment, CLAS is able to investigate the $\pi_{1}(1400)$ through the reaction $\gamma \mathrm{p} \rightarrow \eta \pi^{-} \Delta^{++}$. The analysis of the data is currently underway and preliminary results should soon be made available.

The $\mathrm{f}_{0}(980)$ scalar meson has been interpreted as a multi-quark state ${ }^{\mathbf{1 9}}$ or a kaon anti-kaon bound state ${ }^{20}$ and until recently had never been observed in photoproduction. It is possible that
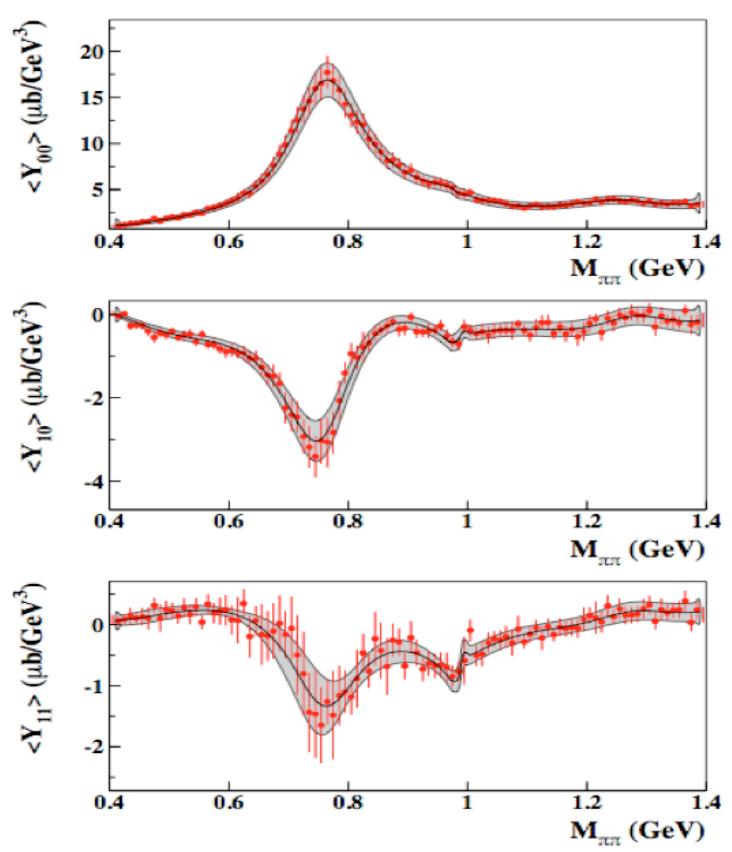

Figure 1: Angular moments. Error bars include the systematic uncertainty related to the photon flux normalization and the moment extraction procedure. The gray band shows the result of the fit of the moments in terms of partial wave amplitudes. the $\mathrm{f}_{0}(980)$, together with the $\mathrm{a}_{0}(980)$, are members of a low-mass nonet of fourquark states. At large distances the quarks may combine into pairs of pseudoscalar mesons. A recent CLAS experiment ${ }^{21}$ has looked at the exclusive photoproduction of $\mathrm{f}_{0}(980)$ mesons on protons with photon energies between 3.0 and $3.8 \mathrm{GeV}$ and momentum transfer in the range $0.4<-\mathrm{t}<1.0 \mathrm{GeV}^{2}$. In this kinematic range the integrated luminosity was about $20 \mathrm{pb}^{-1}$. The resonance was detected via its decay in the $\pi^{+} \pi^{-}$channel by performing a partial wave analysis of the reaction $\gamma p \rightarrow p \pi^{+} \pi^{-}$. The reaction was isolated by detecting the $\pi+$ and proton in CLAS, and reconstructing the $\pi-$ from the missing-mass. Moments of the dipion decay angular distributions were derived from the experimental data. The dominant features in the moments analysis is the $\rho(770)$ in the P-wave, and evidence of the $f_{0}(980)$ in the $Y_{10}$ and $Y_{11}$ moments, through interference between the S-wave and $\mathrm{P}$-wave (Figure 1). The S-wave differential cross section integrated in the mass range of the $\mathrm{f}_{0}(980)$ meson was found to be a factor of about 50 smaller than the cross section for the $\rho$ 
meson. This is the first time the $\mathrm{f}_{0}(980)$ meson has been measured in a photoproduction experiment.

\section{Conclusions.}

The study of the modifications of the properties of hadrons in the medium and the search for exotic mesons are two very active areas of research in hadronic physics. The many ongoing theoretical and experimental studies attest to the vibrancy of these fields. QCD is the fundamental theory of the strong interactions and a lot of progress has been achieved in trying to describe nuclear and hadronic physics in terms of the fundamental degrees of freedom (quarks and gluons). These studies will be pursued through different experiments at JLab in Halls $\mathrm{B}$ and $\mathrm{D}$, taking advantage of the $12 \mathrm{GeV}$ upgrade.

\section{References}

[1] J. J. Dudek et al., arXiv:1004.4930v1 [hep-ph] (2010).

[2] R.S. Hayano and T. Hatsuda, arXiv:0812.1702v2 [nucl-ex] (2009).

[3] S. Leupold, V. Metag and U. Mosel, arXiv:0907.2388v1 [nucl-th] (2009).

[4] T. Hatsuda and S.H. Lee, Nucl. Phys. B 394 (1993) 221.

[5] R. Rapp et al., Eur. Phys. Jour. A 6 (1999) 415.

[6] R. Nasseripour et al., Phys. Rev. Lett. 99, (2007) 262302.

[7] M.H. Wood et al., Phys. Rev. C 78, (2008) 015201.

[8] D. Adamová et al., arXiv:nucl-ex/0611022 v1 13.

[9] S Damjanovic et al., Nucl. Phys. A783 (2007) 327.

[10] M. Wood et al., arXiv:1006.3361v1 [nucl-ex], 17 June, 2010.

[11] N. Isgur et al., Phys. Rev. Lett. 54, (1985) 869.

[12] D. R.Thompson et al., Phys. Rev. Lett. 79, (1997) 1630.

[13] Crystal Barrel Collaboration: A. Abele et al., Phys. Lett. B423, (1998) 175.

[14] G. S. Adams et al., Phys. Rev. Lett. 81, (1998) 5760.

[15] A. Dzierba et al., Phys.Rev.D73, (2006) 072001.

[16] COMPASS Collaboration: A. Alekseev, et al., arXiv:0910.5842v3 [hep-ex] (2010).

[17] G. T. Condo et al. Phys. Rev. D 43, (1991) 2787.

[18] M. Nozar et al., Phys. Rev. Lett. 102, (2009) 102002.

[19] R. A. Jaffe, Phys. Rev. D15, (1977) 267,

M. Alford and R. L. Jaffe, Nucl. Phys. B578 (2000) 367,

L. Maiani et al., Phys. Rev. Lett. 93, (2004) 212002.

[20] J. Weinstein and N. Isgur, Phys. Rev. D41 (1990) 2236.

[21] M. Battaglieri et al., Phys. Rev. Lett. 102, (2009) 102001. 